\title{
Estabilidad a la congelación-descongelación de emulsiones o/w preparadas con aislados de soja nativos y desnaturalizados con diferente solubilidad
}

\author{
Palazolo, G. G. ${ }^{(1)}$, Wagner, J. R. ${ }^{(1)}$ \\ (1) Laboratorio de Propiedades Funcionales de Alimentos, Departamento de Ciencia y Tecnología, Universidad Nacional \\ de Quilmes, Bernal, Argentina - CONICET. \\ Contacto: gpalazolo@unq.edu.ar \\ Recibido: 25/06/2010 - Aprobado: 06/12/2010
}

\begin{abstract}
$\underline{\text { Resumen }}$
En este trabajo se evaluó la estabilidad a la congelación de emulsiones modelo aceite en agua (o/w) preparadas con cuatro aislados de soja: dos nativos (ASN-1 y ASN-2, de similar composición pero diferente solubilidad, $>90 \%$ y $\sim 75 \%$, respectivamente) y dos desnaturalizados (ASD-1 y ASD-2), resultantes de calentar los respectivos aislados nativos $\left(90{ }^{\circ} \mathrm{C}, 5\right.$ minutos). Las emulsiones preparadas con dispersiones acuosas al $2 \% \mathrm{p} / \mathrm{v}$ de ASN o ASD y aceite de girasol refinado $(\Phi=$ 0,25), se congelaron a $-20{ }^{\circ} \mathrm{C}$ por 24 horas y luego se descongelaron a $20^{\circ} \mathrm{C}$. La estabilidad se evaluó a partir de medidas de distribución de tamaño de partícula (difracción láser) y aceite liberado (AL, método de dilución del colorante). Las emulsiones preparadas con ASN-2 y ASD-2 resultaron muy inestables después de ser congeladas y descongeladas, resultado que se evidenció por el aumento del tamaño de partícula y un AL $>\mathbf{2 5 \%}$. Al usar el aislado ASN-1 de mayor solubilidad, las emulsiones fueron más estables y se observó un marcado incremento de la estabilidad cuando sus proteínas eran desnaturalizadas (ASD-1), no observándose prácticamente coalescencia ni aceite separado. En conclusión, la solubilidad y la desnaturalización proteica de los aislados de soja son factores decisivos en la estabilidad de emulsiones frente a la congelación-descongelación.

Palabras clave: Aceite liberado, almacenamiento congelado, desestabilización.
\end{abstract}

\begin{abstract}
$\underline{\text { Abstract }}$
The aim of this work was to evaluate the freeze-thaw stability of model $0 / w$ emulsions prepared with four soy isolates: two native (NSI-1 and NSI-2, with similar composition but different protein solubility, $>90 \%$ and $\sim 75 \%$, respectively), and two denatured (DSI-1 and DSI-2), resulting from heating the respective native ones $\left(90{ }^{\circ} \mathrm{C}, 5 \mathrm{~min}\right)$. Emulsions prepared with NSI or DSI aqueous dispersions $(2 \% \mathrm{w} / \mathrm{v})$ and refined sunflower oil $(\Phi=0.25)$ were frozen at $-20{ }^{\circ} \mathrm{C}$ for $24 \mathrm{~h}$ and subsequently thawed at $20^{\circ} \mathrm{C}$. Freeze-thaw stability was evaluated by particle size distribution (laser diffraction) and free oil (FO, dye-dilution technique) measurements. Emulsions prepared with NSI-2 and DSI-2 were highly destabilized after freeze-thawing process, as was demonstrated by the increase of particle size and $\mathrm{FO}>\mathbf{2 5 \%}$. More stable emulsions were obtained with the high soluble sample NSI-1, and a noticeable increase in stability was observed with denaturation of their proteins (DSI-1). The coalescence and oiling off was almost completely inhibited in DSI-1 emulsions. In summary, solubility and denaturation degree of soy protein isolates have a decisive influence on freeze-thaw stability of protein-stabilized emulsions.

Keywords: Oiling off, frozen storage, destabilization.
\end{abstract}

\section{Introducción}

Los aislados proteicos de soja contienen aproximadamente $90 \%$ de proteínas, constituidas mayoritariamente por las glogulinas de reserva 7S ( $\beta$-conglicinina) y 11S (glicinina). La capacidad de los aislados de soja para formar y estabilizar emulsiones o/w se estudió extensamente en trabajos previos (Mitidieri y Wagner, 2002; Palazolo et al., 2004; Palazolo y Wagner, 2007). La conservación por refrigeración de emulsiones o/w con proteínas como único o principal agente emulsificante es posible, dado que son sistemas estables aun cuando la fase lipídica cristalice. Sin embargo, el almacenamiento congelado de estas emulsiones es un tratamiento altamente desestabilizante (McClements, 2004). En ausencia de crioprotectores, la formación de hielo produce la deshidratación del film interfacial, la crioconcentración de componentes en la fase acuosa y un mayor contacto entre gotas, mecanismos que en conjunto promueven la floculación. Si el film interfacial es incapaz de resistir el estrés inducido por la formación del hielo, se produce además coalescencia y posterior separación de aceite luego de la descongelación (McClements, 2004; Ghosh y Coupland, 2006). Se informaron grados variables de desestabilización frente a la congelación/descongelación en emulsiones o/w preparadas con distintas proteínas (soja, lactosuero, caseinato) (Ghosh et al., 
2008), pero en ningún caso se evaluó el efecto del envejecimiento ("aging") de la muestra. El objetivo del trabajo fue justamente evaluar la influencia del envejecimiento o almacenamiento de aislados de soja sobre la estabilidad de emulsiones modelo o/w frente a la congelación.

\section{Materiales y Métodos}

Preparación de los aislados de soja y las dispersiones acuosas: A partir de harina de soja desgrasada y activa (Solae Latin America, SP, Brasil) se preparó un aislado de soja nativo (ASN-1, 90,2\% N×6,25) por solubilización acuosa, precipitación a $\mathrm{pH} 4,5$, solubilización del precipitado a $\mathrm{pH} 7,5$ y posterior liofilización. Por calorimetría diferencial de barrido (DSC) se corroboró el estado nativo de 7S y 11S (Sorgentini y Wagner, 1999). La muestra ASN-1 se almacenó un año a $4{ }^{\circ} \mathrm{C}$ y resultó en la muestra ASN-2, la cual tiene el mismo contenido proteico que la NSI-1 pero un mayor grado de agregación proteica. Se prepararon dispersiones de ASN-1 y ASN-2 al 2\% p/v en buffer fosfato de sodio $10 \mathrm{~mm} \mathrm{pH}$ 7,0 (agitación magnética, dos horas), se calentaron $\left(90^{\circ} \mathrm{C}, 5\right.$ minutos) y se enfriaron en agua-hielo, obteniéndose las muestras totalmente desnaturalizadas ASD-1 y ASD2. En todas las muestras se determinó la solubilidad proteica como el porcentaje de proteína en el sobrenadante (centrifugación $15000 \mathrm{~g}$, 30 minutos de dispersiones $2 \% \mathrm{p} / \mathrm{v}$ ) respecto de la dispersión inicial (Mitidieri y Wagner, 2002).

Preparación de las emulsiones y tratamiento de congelación/ descongelación: Las emulsiones se prepararon por homogeneización de dispersiones acuosas $(2 \% \mathrm{p} / \mathrm{v})$ y aceite refinado de girasol $(\varphi=0,25)$ en dos etapas: 1) obtención de pre-emulsión con un homogeneizador rotor/estator (Ultraturrax T25, $20000 \mathrm{rpm}, 1$ minuto, IKALabortechnik, Alemania) y 2) circulación de la pre-emulsión en un homogeneizador a válvula de alta presión (400 Bar, Panda 2K, Niro Soavi, Parma, Italia). Las emulsiones $(20 \mathrm{ml}$ en frascos plásticos) se congelaron en un freezer a $-20 \pm 1{ }^{\circ} \mathrm{C}$ durante 24 horas y se descongelaron posteriormente a $20^{\circ} \mathrm{C}$. Las curvas de enfriamiento de la parte media de las emulsiones (termocupla tipo K, registrador Lufft Opus CE 10, Alemania) mostraron que el inicio de la congelación ocurrió a los 50 minutos, llegando a los $-20^{\circ} \mathrm{C}$ a las cuatro horas.

Caracterización de las emulsiones: se determinó el diámetro D43 por dispersión estática de luz (Malvern Mastersizer 2000E, Malvern Instruments, Reino Unido) acoplado a una unidad de dispersión (Hydro 2000MU). Antes de la medida, las emulsiones se diluyeron $(1: 1 \mathrm{v} / \mathrm{v})$ con buffer $10 \mathrm{~mm}$ fosfato de sodio $(\mathrm{pH} 7,0)$ en ausencia y en presencia de $1 \% \mathrm{p} / \mathrm{v}$ de SDS (Anton, 2002), lo cual asegura la disociación de flóculos. Las condiciones de medida fueron: velocidad de dispersión $2000 \mathrm{rpm}$, índices de refracción 1,33 y 1,47 para las fases dispersante y dispersa, respectivamente; coeficiente de absorción 0,01 . El grado de floculación ( $\mathrm{GF} \%$ ) de las emulsiones iniciales y el índice de coalescencia (IC \%) inducido por la congelación se calcularon como: GF $(\%)=\left[\mathrm{D}_{4,3+\text { SDS in }}-\mathrm{D}_{4,3-\mathrm{SDS} \text { in }} / \mathrm{D}_{4,3+\mathrm{SDS} \text { in }}\right] \times 100$, IC $(\%)=\left[D_{4,3+\text { SDS c-d }}-D_{4,3+\text { SDS in }} 1 / D_{4,3+\text { SDS in }}\right] \times 100$, donde los subíndices - SDS, + SDS, in y c-d indican valores obtenidos sin y con SDS, antes y después de la congelación/descongelación, respectivamente (Palazolo y Wagner, 2007). El aceite liberado (AL \%) se determinó con el método espectrofotométrico de dilución del colorante liposoluble Sudan III, descripto por Palanuwech et al. (2003) y modificado por Palazolo y Wagner (2007).

\section{Resultados y Discusión}

La solubilidad proteica (SP) de la muestra ASN-1 (93,2 \pm $1,4 \%)$ disminuyó significativamente por efecto del almacenamiento refrigerado (ASN-2, $\mathrm{SP}=74,8 \pm 1,2 \%$ ), lo que se atribuye principalmente a la agregación de la globulina 11S (Palazolo et al., 2004). Este envejecimiento también se vio reflejado en las muestras desnaturalizadas: $\mathrm{SP}=96,1 \pm 1,3 \%$ y $87,5 \pm 1,4 \%$ para ASD- 1 y ASD2 , respectivamente. Al tratar térmicamente una dispersión acuosa de aislado de soja a baja concentración, las globulinas $7 \mathrm{~S}$ y $11 \mathrm{~S}$ se desnaturalizan, disocian y forman agregados solubles, en tanto que los agregados insolubles preexistentes se disocian parcialmente, lo cual incrementa la solubilidad, especialmente evidente en la muestra almacenada ASN-2. El efecto del almacenamiento también se observó en las características de las emulsiones iniciales. La concentración proteica usada $(2 \% \mathrm{p} / \mathrm{v})$ fue suficiente para cubrir el área creada durante la homogeneización, con más del $20 \%$ de proteína no adsorbida en la fase acuosa (Palazolo y Wagner, 2007). Sin embargo, las emulsiones preparadas con las muestras almacenadas dieron valores $\mathrm{D}_{4,3}$ levemente superiores y un notable incremento en el grado de floculación (GF \%) respecto a las de las muestras frescas (Gráfico 1). Estos flóculos se forman por un mecanismo de puenteo, ya que fueron estables en las condiciones de medida del tamaño de partícula. En el caso de las muestras almacenadas, la floculación se atribuiría a una más lenta adsorción de proteína en la interfase por la presencia de agregados y a una menor cantidad de proteína soluble disponible (McClements, 2004). Aunque la desnaturalización térmica induce la formación de agregados solubles, el incremento de la hidrofobicidad superficial de las proteínas favorece la formación de emulsiones con menor GF \% (emulsión ASD-2 respecto a ASN-2, Gráfico 1).

En las emulsiones preparadas con las muestras frescas ASN-1 y ASD-1 se observó una inhibición casi completa de liberación de aceite luego de la descongelación y en particular en la emulsión ADS-1 una mayor estabilidad frente a la coalescencia (Tabla 1). El incremento de la concentración efectiva de proteína en la interfase en al menos un orden de magnitud, sumado a la alta tendencia de la globulina $11 \mathrm{~S}$ nativa a la agregación a bajas temperaturas, hace altamente probable que se agregue en la interfase por efecto de la congelación y perturbe la integridad del film interfacial, induciendo a la coalescencia (Palazolo y Wagner, 2007). Este efecto no es observado cuando la $11 \mathrm{~S}$ está desnaturalizada térmicamente (ASD-1), acorde a la notable disminución de IC \% (Tabla 1). En cambio, las emulsiones preparadas con ASN-2 y ASD-2 mostraron un marcado grado de desestabilización luego del almacenamiento congelado. Al descongelarse se observó separación gravitacional, una fase crema coagulada con formación de agregados proteicos y una capa de aceite en la parte superior de la emulsión, acorde con los altos valores de IC \% (>104) y AL \% (Tabla 1).

En estas emulsiones preparadas con muestras envejecidas, el efecto positivo de la desnaturalización térmica se ve enmascarado (Tabla 1). Cuando la emulsión inicial tiene un mayor grado de floculación, los flóculos tienen menor capacidad de acomodarse en el volumen reducido de la fase acuosa no congelada, desestabilizándose rápidamente por coalescencia. Por la misma razón, la presencia de gotas individuales de mayor tamaño también puede tener una influencia negativa en la estabilidad posterior de la emulsión frente a la congelación.

El efecto del envejecimiento de proteínas sobre la estabilidad frente a la congelación de emulsiones o/w es un factor que no debe subestimarse. Aunque la estabilización total de la emulsión requiere la adición de crioprotectores, es fundamental contar con una emulsión de partida de reducido tamaño de gotas y un bajo grado de floculación, lo 
cual se logra con una adecuada solubilidad y actividad superficial del emulsionante. Este efecto podría ser particularmente importante en la preparación de emulsiones con aislados de soja comerciales, que en la mayoría de los casos tienen una solubilidad significativamente inferior a la de aquellos preparados en el laboratorio

\begin{tabular}{|c|c|c|}
\hline Muestras & IC (\%) & AL (\%) \\
\hline ASN-1 & $>10^{4}$ & $1,7 \pm 0,3$ \\
\hline ASD-1 & $85 \pm 1$ & $1,6 \pm 0,4$ \\
\hline ASN-2 & $>10^{4}$ & $31,2 \pm 3,0$ \\
\hline ASD-2 & $>10^{4}$ & $33,7 \pm 0,7$ \\
\hline
\end{tabular}

Tabla 1. Parámetros de desestabilización de emulsiones o/w preparadas con aislados nativos y desnaturalizados de soja (ASN y ASD, respectivamente) y sometidas a congelación-descongelación

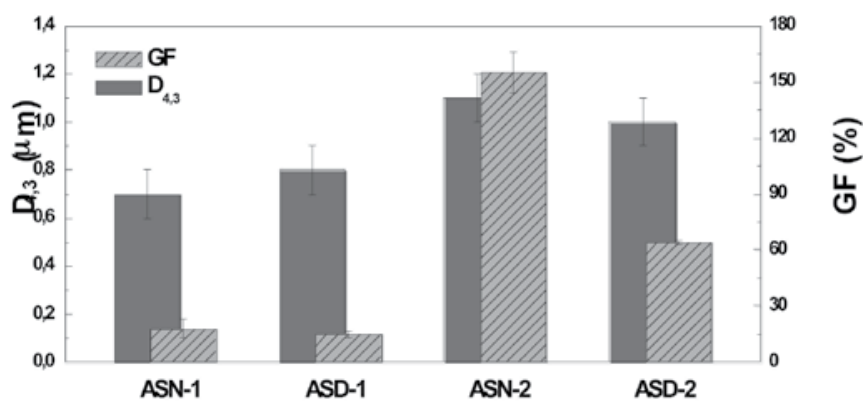

Gráfico 1. Diámetros promedio D4,3 de gotas individuales y grado de floculación (GF \%) de emulsiones o/w preparadas con aislados nativos (ASN) y desnaturalizados (ASD) de soja. Los valores D4,3 se obtuvieron a partir de la distribución de tamaño de partícula en presencia de SDS.

\section{Referencias}

- ANTON, M.; BEAUMAL, V.; BROSSARD, C.; LLAMAS, G.; LE DENMAT, M. Droplet flocculation and physical stability of oil-inwater emulsions prepared with hen egg yolk. En: ANTON, M. Food emulsions and dispersion. Trivandrum: Research Sinpost, 2002. pp. 15-28.

- McCLEMENTS, D.J. Protein-stabilized emulsions. En: Current Opinion in Colloid and Interface Science. 2004, (9):305-313.

- MITIDIERI, F.E.; WAGNER, J.R. Coalescence of o/w emulsions stabilised by whey and isolate soybean proteins. Influence of thermal denaturation, salt addition and competitive interfacial adsorption En: Food Research International. 2002, 35(6):547-557.

- PALANUWECH, J; PONITENI, R; ROBERTS, R.F.; COUPLAND, J.N. A method to determine free fat in emulsions. En: Food Hydrocolloids. 2003, 17(1):55-62.

- PALAZOLO, G.G.; WAGNER, J.R. Effect of freezing on stability of soy protein and sodium caseinate oil-in-water emulsions. En: LUPANO, C.E. Functional properties of food components. Kerala: Research Signpost, 2007. pp. 33-56.

- PAlazOlO, G.G.; SORGENTINI, D.A.; WAGNER, J.R. Emulsifying properties and surface behavior of native and denatured whey soy proteins in comparison with other proteins. Creaming stability of o/w emulsions. En: Journal of the American Oil Chemists 'Society. 2004, 81(7):625-632.

- GHOSH, S; COUPLAND, J.N. Factors affecting the freeze-thaw stability of emulsions. En: Food Hydrocolloids. 2008, 22(1):105111 .
- GHOSH, S.; CRAMP, G.; COUPLAND, J.N. Effect of aqueous composition on the freeze-thaw stability of emulsions. En: Colloids and Surfaces A: Physicochemical and Enginnering Aspects. 2006, 272(1-2):82-88.

- SORGENTINI, D.A.; WAGNER, J.R. Comparative study of structrural characteristics ant thermal behavior of whey and isolate soybean proteins. En: Journal of Food Biochemistry. 1999, 23(5):489-507. 